\title{
Efeitos da Insuficiência de Água sobre a Produtividade de Empresas Brasileiras
}

\author{
Welber Tomás de Oliveira Carlos César Santejo Saiani \\ PPGE-UFU \\ IERI-UFU \\ weelber@live.com \\ ssaiani@ufu.br \\ Ana Paula Macedo de Avellar \\ IERI-UFU \\ anaavellar@ufu.br
}

\begin{abstract}
Resumo
O objetivo deste estudo é investigar, empiricamente, se a insuficiência de água para a produção influencia a produtividade do trabalho e a produtividade total dos fatores (PTF) de empresas manufatureiras brasileiras com informações referentes a 2007 disponibilizadas pela Investiment Climate Survey do Banco Mundial. Para calcular a PTF, é adotada uma metodologia proposta pela literatura. Para mensurar os efeitos médios da insuficiência de água para a produção sobre a produtividade são adotados métodos de estimações por Propensity Score Matching para lidar com possível viés de auto seleção e balancear, em termos de atributos observados, os grupos de tratamento (empresas com insuficiência de água) e controle. Os resultados das diferentes estimações permitem interpretar, com certa robustez, como causal o efeito médio negativo associado à insuficiência de água sobre a PTF nas empresas que passaram por tal problema.
\end{abstract}

Palavras-chave: Produtividade; PTF; Insuficiência de Água; Propensity Score Matching.

\begin{abstract}
This article aims to investigate empirically the effect of water insufficiency for production on labor productivity and total factor productivity (TFP) of Brazilian manufacturing firms with information for 2007 provide by the World Bank's Investiment Climate Survey (ICS). For the measurement of PTFs the study adopts a methodology based in the literature. To measure the average effects of water insufficiency on productivity, Propensity Score Matching estimation methods are used to deal with possible self-selection bias and to balance in terms of observed attributes the treatment (firms with water insufficiency) and control groups. The results of the different estimations allow us to interpret, with a certain robustness, as causal the negative average effect associated to the water insufficiency on the firms' TFP.
\end{abstract}

Keywords: Productivity; TFP; Water Insufficiency; Propensity Score Matching.

Classificação JEL: D24, L60, L95

Área ABEIN: Área 1 - Indústria e competitividade;

1.3 Crescimento, produtividade e competitividade 


\section{Introdução}

O objetivo deste estudo é investigar, empiricamente, se a insuficiência de água para a produção influencia a produtividade do trabalho e a produtividade total dos fatores (PTF) de empresas brasileiras. Parte-se das premissas de que a água é um importante insumo de produção nos mais diversos setores e que problemas no abastecimento de água podem afetar a saúde dos trabalhadores e, consequentemente, suas produtividades no trabalho e, também, os processos produtivos das empresas, diminuindo a eficiência no uso de capital e insumos intermediários.

Para atingir o objetivo proposto, são utilizadas informações de empresas manufatureiras brasileiras referentes a 2007 disponibilizadas pela Investiment Climate Survey (ICS) do Banco Mundial. Por meio destas informações, são mensuradas as produtividades do trabalho e as PTFs das firmas com dados disponíveis. Para o cálculo das PTFs, é adotada uma metodologia recomendada pela literatura, inclusive para a mesma base de dados. Já para mensurar os efeitos médios da insuficiência de água são utilizados métodos de estimação por Propensity Score Matching para lidarem com a possibilidade de viés de auto seleção e balancearem, em termos de atributos observados, os grupos de tratamento (com insuficiência de água) e de controle.

Antecipadamente, é importante apontar que os resultados encontrados permitem atribuir, com relativa robustez, causalidade às reduções médias estimadas da PTF devido à insuficiência de água nas firmas manufatureiras da amostra que passaram por tal problema em 2007. Já no caso da produtividade do trabalho, as evidências obtidas sinalizam que não existem efeitos.

Tais evidências contribuem para o preenchimento de uma lacuna na literatura empírica para o caso de empresas brasileiras e, ao mesmo tempo, ressaltam dois desafios históricos para as políticas públicas brasileiras: incentivar a produtividade das empresas e, para isso e outros objetivos, investir em infraestrutura, em especial, no abastecimento de água. $\mathrm{O}$ aumento da produtividade - que, no Brasil, é aquém do necessário -, segundo a literatura, pode estimular o crescimento econômico com inclusão social e reduções de desigualdades sociais e regionais.

Além desta introdução e das considerações finais, o estudo é dividido em três seções. Na segunda, é realizada uma revisão da literatura e são apresentados alguns dados para ilustrar a relação entre a produtividade das empresas e o abastecimento de água e como estas questões se configuram como desafios importantes para o Brasil. Na terceira, são discutidos as estratégias empíricas e os dados utilizados nas estimações. Na quarta, os resultados são analisados.

\section{Produtividade e abastecimento de água: relação e desafios para o Brasil}

A produtividade dos fatores de produção tem papel central no debate econômico, pois a literatura aponta efeitos no crescimento econômico, inclusão social e redução de desigualdades (DE NEGRI; CAVALCANTE, 2014a; MUSSOLINI; TELES, 2010). No Brasil, estes desafios coexistem, o que torna a produtividade ainda mais central, inclusive pelas evidências recentes não favoráveis. Os trabalhos organizados no livro de De Negri e Cavalcante (2014b) estimam a produtividade do trabalho e a PTF no Brasil nas últimas décadas, não deixando dúvidas que, independentemente da medida e do método, a produtividade é baixa e cresce aquém do ideal.

Ellery Júnior (2014) identifica trajetória comum nas estimativas: a produtividade cresceu de forma consistente no início e irregular no final dos anos 1970, caiu significativamente nos anos 1980, seguida por pequena recuperação nas décadas de 1990 e 2000 . De forma geral, a produtividade cresceu, de 1970 a 2011, a $1 \%$ ao ano, taxa muito baixa. Mesmo em um período favorável, 2003 a 2011, o aumento da produtividade foi baixo -2,6\%. Miguez e Moraes (2014) estimam que se os setores brasileiros tivessem produtividades similares às dos mesmos setores dos Estados Unidos e da Alemanha, a produtividade brasileira seria de $428 \%$ a $577 \%$ superior. 
Ganhos de produtividade podem ser obtidos pela convergência de vários fatores, como progresso técnico e maior eficiência. O primeiro é o deslocamento da fronteira tecnológica, no geral, por inovações; o segundo é a aproximação desta fronteira. Na base desta cadeia causal, emergem diversos aspectos inter-relacionados que podem incentivar inovações e eficiência, como instituições que protejam os direitos de propriedade, reduzindo custos de transação, e investimentos em capitais humano e físico (e estoques), destacando-se, em relação aos físicos, as infraestruturas econômicas (DE NEGRI; CAVALCANTE, 2014b; MUSSOLINI; TELES, 2010). No Brasil, estas são um grande desafio, pois persistem sérios gargalos (IPEA, 2010).

Banco Mundial (1994) define infraestruturas econômicas como atividades com atributos técnicos e econômicos semelhantes - por exemplo: intensivas em capital, demandas inelásticas, externalidades positivas, elevadas economias de escala e de escopo e sunk costs que impõem, em alguns casos, limites à concorrência. Pêgo Filho (1999), seguindo tal definição, classifica atividades e serviços de infraestrutura econômica em: i) serviços de utilidade pública - como: energia elétrica, telecomunicação, captação e distribuição de gás e serviços de saneamento básico, dentre os quais, o abastecimento de água -; ii) serviços públicos (ou obras públicas) rodovias e drenagem; e iii) outros setores de transporte - como: portos, ferrovias e aeroportos.

$\mathrm{Na}$ literatura, há evidências favoráveis à existência de efeitos positivos dos investimentos em infraestrutura econômica (ou estoque) no crescimento econômico. No geral, são defendidos três efeitos: i) aumento direto da demanda agregada, estimulando produção (RIGOLON, 1998); ii) redução de custos unitários dos insumos (MORRISON; SCHWARTZ, 1996); e iii) elevações das produtividades dos insumos e da PTF. Para evidências deste efeito, inclusive para o Brasil, ver: Aschauer (1989), Barro (1990), Munnel (1990), Easterly e Rebelo (1993), Ferreira (1996), Ferreira e Malliagros (1997), Cruz et al. (2010) e Mussolini e Teles (2010), entre outros.

Em relação aos serviços de saneamento básico e, mais especificamente, ao abastecimento de água, alguns trabalhos discutem e/ou apresentam evidências de seus efeitos no crescimento econômico, principalmente via produtividade dos fatores. No trabalho de Aschauer (1990), por exemplo, é constatado que gastos públicos com as utilidades públicas (ou seus estoques), como o abastecimento de água, estimularam ganhos de produtividade e o crescimento nos Estados Unidos. Evidências nesse sentido também são obtidas por, entre outros: Uchimura e Gao (1993) para Taiwan e Coreia; Bregman e Marom (1999) para Israel; Mastromarco e Woitek (2006) para a Itália; Rigolon (1998), Mussolini e Teles (2010) e Schettini e Azzoni (2015) para o Brasil.

No nível das firmas, alguns trabalhos utilizam a mesma base de dados do presente estudo - Investiment Climate Survey (ICS) do Banco Mundial. Escribano e Guasch (2008), Escribano et al. (2008, 2010), Fajnzylber (2009) e Moyo (2011) obtêm relações significativas e positivas entre variáveis de infraestrutura econômica em geral, inclusive de disponibilidade hídrica para a produção, e a PTF na Turquia e em países da América Central, América Latina e África.

Por um lado, a disponibilidade de água pode influenciar a PTF por ser este um importante insumo para a produção de diversos produtos, inclusive manufaturados. Nesse sentido, ANA (2017) apresenta parâmetros de uso de água em vários setores de atividades manufatureiras. Deve-se considerar, ainda, que se a água estiver disponível, mas com qualidade inapropriada para o uso, as firmas devem alocar recursos para tal adequação, o que afeta suas produtividades.

Por outro lado, acesso e qualidade da água podem afetar a produtividade do trabalho. No longo prazo, crianças que contraíram doenças de veiculação hídrica ou sofreram privações de consumo de água podem ter desenvolvimentos físico e intelectual comprometidos, afetando seus desempenhos escolares. Problemas no desenvolvimento e na formação de capital humano influenciam, no futuro, suas produtividades no trabalho. No curto prazo, trabalhadores que contraem doenças de veiculação hídrica ou não consomem água em níveis adequados, também têm suas produtividades prejudicadas (CVJETANOVIC, 1986; HELLER, 1997; IBRE, 2010).

Apesar do Brasil ter relativa abundância de recursos hídricos, o abastecimento de água para consumo e produção também é um importante desafio. Historicamente, investimentos em 
infraestruturas de captação, distribuição e tratamento de água foram aquém dos necessários, devido a uma aparente desmotivação política, à persistência de restrições e à ineficiência da maioria dos provedores (TUROLLA, 2002; TONETO JÚNIOR; SAIANI, 2006). Os resultados dos baixos investimentos são: i) indisponibilidade hídrica e/ou racionamentos em vários locais; ii) déficits de acesso distribuídos de forma desigual pelo país; iii) perdas na distribuição; e iv) problemas de qualidade da água ofertada (ANA, 2010; IBGE, 2011; SAIANI et al., 2015).

\section{Estratégias empíricas e dados}

Para avaliar se a insuficiência de água para produção afeta a produtividade do trabalho e a PTF das empresas brasileiras, utiliza-se a base de dados Investiment Climate Survey (ICS) do Banco Mundial. No cálculo da produtividade do trabalho e da PTF, adota-se as sugestões de Ellery Júnior (2014), Messa (2014) e Escribano e Guasch (2008). Os dois primeiros trabalhos discutem os métodos mais comuns de cálculo e estimação; o terceiro debate métodos e variáveis para a realização de estimações de efeitos sobre a produtividade com a base de dados ICS.

A sugestão é que a produtividade do trabalho da empresa $i\left(\operatorname{Prod}_{i}\right)$ seja mensurada pela razão entre vendas $V_{i}$ (unidades monetárias) - proxy para o produto - e número de trabalhadores $L_{i}$ (ou horas trabalhadas), sendo aplicada uma transformação logarítmica - logaritmo natural $(\ln )$. Ou seja: $\ln \left(\operatorname{Prod} L_{i}\right)=\ln \left(V_{i} / L_{i}\right)$. Pode-se argumentar que a variável vendas não é uma proxy apropriada para os produtos, pois não discrimina quantidades e preços. No entanto, Messa (2014) sustenta que, em estimações ao nível da empresa, esta utilização é comum e válida, pois é difícil ter dados disponíveis de preço e quantidade para todas as firmas. Ademais, na proposta Escribano e Guasch (2008), que fundamenta este estudo, as vendas são a proxy para o produto.

A produtividade do trabalho refere-se apenas a um fator de produção, o que incentiva o uso da PTF e comparações entre elas. A PTF não tem cálculo direto, sendo que sua mensuração depende de alguns pressupostos: i) adoção de uma função produção; ii) plena substituição dos fatores; e iii) retornos constantes. Usualmente, é adotada a função Cobb-Douglas $V_{i}=A_{i} K_{i}^{\alpha} L_{i}^{\beta}$, sendo $V_{i}$ o produto da firma $i, K_{i}$ o estoque de capital físico, $L_{i}$ a quantidade de trabalho e $A_{i}$ a PTF. Assim, $A_{i}=V_{i} /\left(K_{i}^{\alpha} L_{i}^{\beta}\right)$. Em estimações econométricas com variáveis em logaritmo, $A_{i}$ é o resíduo. Nesse sentido, Escribano e Guasch (2008) propõe a adoção de uma Cobb-Douglas expandida por insumos intermediários $\left(M_{i}\right)$ para estimar a PTF de acordo com a equação (1).

$\ln \left(V_{i}\right)=\theta+\alpha \ln \left(K_{i}\right)+\beta \ln \left(L_{i}\right)+\delta \ln \left(M_{i}\right)+\varepsilon_{i}$

sendo: $V_{i}$ as vendas da empresa $i$ - variável dependente -; $\theta$ a constante; $K_{i}$ o estoque de capital - valor líquido de todos ativos fixos (unidades monetárias) -; $\alpha$ o coeficiente associado ao capital; $L_{i}$ o trabalho - trabalhadores permanentes; $\beta$ o coeficiente associado ao trabalho; $M_{i}$ o custo total dos insumos intermediários (unidades monetárias) ${ }^{1} ; \delta$ o vetor de coeficientes associados aos insumos intermediários; e $\varepsilon_{i}$ é o resíduo - o logaritmo da PTF $\left(\ln \left(A_{i}\right)\right)$.

Vale destacar que a base de dados ICS é constituída por dados referentes a 2007 para uma amostra de 1.802 empresas brasileiras. As informações da base foram coletadas, pelo Banco Mundial, por meio de questionários nos quais as empresas respondiam várias questões, mas não eram obrigadas a isso, o que faz com que o número de respostas se altera segundo a questão. Considerando os dados necessários para produtividade do trabalho, PTF, insuficiência de água e insumos intermediários, neste estudo, são consideradas as duas amostras apresentadas na Tabela 1, distribuídas segundo as variáveis e as unidades federativas brasileiras. Há dados para a produtividade do trabalho e a suficiência de água para a produção para 982 firmas (amostra

\footnotetext{
${ }^{1}$ Custo total de todos os materiais intermediárias (inputs) utilizados na atividade produtiva, como: matérias-primas, energia elétrica, água e combustível. Esta é a variável proposta por Escribano e Guasch (2008).
} 
I), 60 com insuficiência $(6,1 \%)$ e 922 sem $(93,9 \%)$. Já para o cálculo da PTF e a suficiência de água, a amostra é menor: 743 firmas (amostra II), 44 com insuficiência $(5,9 \%)$ e $699(94,1 \%)$ sem. Na amostra II não há dados para o cálculo da PTF referentes a firmas de Pernambuco.

Tabela 1 - Distribuições das amostras de empresas, segundos as variáveis e unidades federativas (2017)

\begin{tabular}{c|c|c|c|c}
\hline $\begin{array}{c}\text { Unidades Federativas / } \\
\text { Variáveis }\end{array}$ & Produtividade do Trabalho (amostra I) & \multicolumn{2}{|c}{ PTF (amostra II) } \\
\cline { 2 - 5 } & Com Insuficiência & Sem Insuficiência & Com Insuficiência & Sem Insuficiência \\
\hline Amazonas & 3 & 8 & 3 & 7 \\
Bahia & 4 & 49 & 3 & 41 \\
\hline Ceará & 3 & 31 & 3 & 25 \\
Distrito Federal & 0 & 6 & 0 & 5 \\
\hline Goiás & 1 & 43 & 1 & 34 \\
\hline Mato Grosso & 6 & 12 & 3 & 8 \\
Minas Gerais & 5 & 106 & 5 & 89 \\
\hline Paraíba & 0 & 13 & 0 & 9 \\
\hline Paraná & 7 & 92 & 4 & 73 \\
\hline Pernambuco & 1 & 22 & 0 & 0 \\
\hline Rio de Janeiro & 7 & 80 & 6 & 72 \\
Rio Grande do Sul & 2 & 135 & 2 & 716 \\
\hline Santa Catarina & 8 & 98 & 8 & 76 \\
\hline São Paulo & 13 & 227 & 6 & $\mathbf{6 9 9}$ \\
\hline Total & $\mathbf{6 0}$ & $\mathbf{9 2 2}$ & $\mathbf{4 4}$ & 144 \\
\hline
\end{tabular}

Fonte: Banco Mundial, ICS. Elaboração própria.

A Tabela 2 apresenta as distribuições das amostras por setores de atividades, segundo classificação da própria ICS. Observa-se que, apesar da base também considerar serviços, não há observações para empresas deste setor com todos os dados necessários. Por isso, o escopo deste estudo acaba sendo as manufaturas. Vale apontar que, dos 16 setores da amostra total da pesquisa, restam apenas 9 para produtividade do trabalho e 8 para a PTF, com a perda do setor de têxteis na última. Considerando discussões de ANA (2017), os setores considerados nas análises são "tradicionais", com forte importância na economia brasileira, e estão entre os principais consumidores de água, em especial alimentos, químicos, sapatos e couro e têxteis.

Tabela 2 - Distribuições das amostras de empresas, segundo as variáveis e os setores de atividades (2017)

\begin{tabular}{c|c|c|c|c}
\hline Unidades Federativas / & \multicolumn{2}{|c|}{ Produtividade do Trabalho } & \multicolumn{2}{|c}{ PTF } \\
\cline { 2 - 5 } Variáveis & Com Insuficiência & Sem Insuficiência & Com Insuficiência & Sem Insuficiência \\
\hline Alimentos & 15 & 89 & 12 & 81 \\
\hline Autopeças & 5 & 97 & 2 & 79 \\
Máquinas e Equipamentos & 7 & 154 & 6 & 128 \\
\hline Móveis & 9 & 126 & 8 & 104 \\
Químicos & 13 & 96 & 10 & 82 \\
Sapatos e Couro & 2 & 113 & 1 & 104 \\
Têxteis & 1 & 103 & 0 & 0 \\
Vestuário & 7 & 134 & 4 & 9 \\
\hline Outras Manufaturas & 1 & 10 & 1 & $\mathbf{6 9 9}$ \\
\hline Total & $\mathbf{6 0}$ & $\mathbf{9 2 2}$ & $\mathbf{4 4}$ & $\mathbf{6}$ \\
\hline
\end{tabular}

Fonte: Banco Mundial, ICS. Elaboração própria.

Para os domicílios, Rezende et al. (2007) defendem que o acesso ao abastecimento de água depende, pelo lado da demanda, da disposição e capacidade de pagamento (tarifas e gastos com a conexão às redes) e, pelo lado da oferta, da existência e qualidade da provisão. É plausível supor que o mesmo vale para as empresas. Portanto, a insuficiência de água para a produção, declarada por algumas firmas no questionário da ICS, depende de decisões dos provedores de abastecimento de água e das firmas, que optam por se conectarem às redes, investindo nesse 
sentido e pagando tarifas. Se a opção for pela não conexão ou na ausência de redes, as firmas podem buscar fontes alternativas - como poços, nascentes e aproveitamento da chuva - ou até mudarem suas plantas para locais que atendam melhor suas necessidades. Nas amostras I e II, cerca de $72 \%$ das firmas declararam que usavam água oriunda somente de "fontes públicas" 2 .

Portanto, a ocorrência de insuficiência de água pode ser, em algum grau, influenciada por atributos das empresas, que também determinam suas produtividades (do trabalho e total). É possível, assim, que as produtividades das empresas com insuficiência de água sejam distintas das demais independentemente da indisponibilidade de água. Assim, existiria um viés de auto seleção. Se a insuficiência de água for considerada como um "tratamento", pode-se interpretar que a seleção ao tratamento não é independente de seus resultados potenciais (produtividades).

Baseando-se no modelo Roy-Rubin de resultados potenciais (ROY, 1951; RUBIN, 1974), para mensurar os efeitos médios da insuficiência de água sobre a produtividade do trabalho e a PTF, o ideal seria observar, concomitantemente, os resultados potenciais da empresa $i$ nas situações de insuficiência $\left(Y_{i}^{1}\right)$ e de suficiência de água $\left(Y_{i}^{0}\right)$. Se isso fosse possível, o efeito médio do tratamento sobre os tratados $\left(\tau_{A T T}\right)^{3}$ poderia ser calculado pela equação $(2)^{4}$.

$\tau_{A T T}=E\left[\left(Y_{i}^{1}-Y_{i}^{0}\right) \mid A_{i}=1\right]=E\left[Y_{i}^{1} \mid A_{i}=1\right]-E\left[Y_{i}^{0} \mid A_{i}=1\right]$

sendo: $A_{i}$ a variável binária representativa do status do tratamento (igual a 1 se a empresa $i$ teve insuficiência de água para produção em 2007); $Y_{i}^{1} \mid A_{i}=1$ o resultado da insuficiência de água na empresa $i$ que efetivamente sofreu tal insuficiência; e $Y_{i}^{0} \mid A_{i}=1$ o resultado potencial da suficiência de água (não tratamento) na empresa $i$ que passou por insuficiência de água.

O problema é que, em dado momento, apenas um dos resultados é passível de observação: i) o resultado da insuficiência de água nas firmas que passaram por este problema $\left(Y_{i}^{1} \mid A_{i}=1\right)$; e ii) o resultado da suficiência de água nas firmas que não tiveram restrições de disponibilidade de água $\left(Y_{i}^{0} \mid A_{i}=0\right)$. Assim, o que é possível mensurar é a diferença média desses resultados: $E\left[Y_{i}^{1} \mid A_{i}=1\right]-E\left[Y_{i}^{0} \mid A_{i}=0\right]$. Somando e dividindo, na expressão, o resultado potencial da suficiência nas firmas que passaram por insuficiência $\left(E\left[Y_{i}^{0} \mid A_{i}=1\right)\right.$, obtém-se a equação (3).

$\underbrace{E\left[Y_{i}^{1} \mid A_{i}=1\right]-E\left[Y_{i}^{0} \mid A_{i}=0\right]}_{\text {Efeito Observado }}=\underbrace{E\left[\left(Y_{i}^{1}-Y_{i}^{0}\right) \mid A_{i}=1\right]}_{\tau_{A T T}}+\underbrace{E\left[Y_{i}^{0} \mid A_{i}=1\right]-E\left[Y_{i}^{0} \mid A_{i}=0\right]}_{\text {Viés de Auto Seleção }}$

Com viés de auto seleção, o resultado da não participação no tratamento é diferente entre as empresas tratadas (com insuficiência de água) e não tratadas (sem insuficiência de água), de modo as não tratadas não são um bom contrafactual para as tratadas. Assim, a diferença dos resultados médios entre elas é uma medida inadequada do efeito médio $\left(\tau_{A T T}\right)$ do tratamento. Se a opção pelo tratamento fosse aleatória (não correlacionada a atributos que influenciam suas consequências), os resultados potencias da suficiência de água seriam os mesmos para empresas com ou sem insuficiência. Consequentemente, o viés de auto seleção seria nulo e as não tratadas seriam um contrafactual para as tratadas. Porém, não é plausível supor a aleatorização ${ }^{5}$.

Diante destes fatos, para avaliar o efeito da insuficiência de água sobre a produtividade, é necessário um método de estimação que lide com o viés de auto seleção. Para dados em crosssection, a opção é um método baseado na seleção por atributos observados, assumindo a hipótese de ignorabilidade do tratamento ${ }^{6}$. Ou seja, condicionais a um conjunto de atributos observados da firma $i\left(X_{i}\right)$, que determinam a seleção, os resultados potenciais (produtividades)

\footnotetext{
${ }^{2}$ Pela definição da ICS, água originária de serviços públicos, poços públicos e outras fontes públicas.

${ }^{3}$ Em inglês, average treatment effect on the treated (ATT).

${ }^{4}$ Assumindo a hipótese de que o resultado potencial de uma empresa independe do status do tratamento em outras empresas - stable unit treatment value assumption (SUTVA). Para mais detalhes, ver: Cox (1958) e Rubin (1978).

${ }^{5}$ Para discussões mais detalhadas sobre os benefícios da aleatorização (randomização) e os problemas da ausência de um processo deste tipo, conferir: Rubin (1974), Smith e Todd (2005) e Duflo et al. (2006), entre outros.

${ }^{6}$ Ignorability (unconfoundedness) assumption. Ver: Rubin (1977) e Firpo e Pinto (2013).
} 
independem do status do tratamento. Respeitando tal condição, o viés de seleção desaparece. Assim, $E\left[Y_{i}^{0} \mid X_{i}, A_{i}=0\right]$ é um contrafactual adequado para $E\left[Y_{i}^{1} \mid X_{i}, A_{i}=1\right]$ e o efeito médio da insuficiência de água $\left(\tau_{A T T_{X}}\right)$ nos tratados pode ser calculado de acordo com a equação (4).

$\tau_{A T T_{X}}=E\left[\left(Y_{i}^{1}-Y_{i}^{0}\right) \mid X_{i}, A_{i}=1\right]=E\left[Y_{i}^{1} \mid X_{i}, A_{i}=1\right]-E\left[Y_{i}^{0} \mid X_{i}, A_{i}=0\right]$

Para estimações baseadas em (4), é importante que os grupos de controle e de tratamento sejam similares, o que também é bastante relevante neste estudo em função do pequeno número de firmas tratadas relativamente às não tratadas nas amostras (Tabelas 1 e 2). Maior semelhança entre os grupos pode ser garantida por meio de uma estratégia de matching (pareamento) que identifique um grupo de controle a partir do conjunto $X$ de atributos observados das empresas. Contudo, quanto maior o número de atributos considerados, maior a dificuldade do matching.

É possível lidar com tal problema com um método de Propensity Score Matching (PSM), no qual o paramento é feito pela probabilidade predita (propensity score) da empresa $i$ sofrer insuficiência de água (participar do tratamento), considerando atributos observados $X_{i}$. Para Rosenbaum e Rubin (1983), se, condicionais a $X_{i}$, os resultados são independentes do status do tratamento, a independência também é observada nos resultados condicionais aos propensity scores $P\left(X_{i}\right)$. Estes, estimados por Logit ou Probit ${ }^{7}$, são positivos e os atributos observados $X_{i}$ não necessitam determinar perfeitamente o status do tratamento - ou seja, $0<P\left(X_{i}\right)<1$.

Para garantir maior comparabilidade entre os grupos de controle e tratamento, pode-se impor uma condição de suporte comum, retirando do primeiro grupo firmas com probabilidades preditas inferiores à mínima ou superiores à máxima das tratadas ${ }^{8}$. Considerando a hipótese de ignorabilidade do tratamento e a condição de suporte comum ${ }^{9}$, a equação (5) mostra a maneira segundo a qual o efeito médio condicionado aos propensity scores $\left(\tau_{A T T_{X}}^{P S M}\right)$ pode ser calculado.

$\tau_{A T T_{X}}^{P S M}=E\left[\left(Y_{i}^{1}-Y_{i}^{0}\right) \mid P\left(X_{i}\right), A_{i}=1\right]=E\left[Y_{i}^{1} \mid P\left(X_{i}\right), A_{i}=1\right]-E\left[Y_{i}^{0} \mid P\left(X_{i}\right), A_{i}=0\right]$

O PSM é uma estratégia de reponderação das observações para balancear as distribuições dos atributos observados entre os grupos de tratamento e de controle. Os pesos dependem do método para o matching. Há vários métodos, que se diferem quanto às formas pelas quais são definidos grupos de controle e pesos, sendo que, assintoticamente, os resultados são próximos e não é possível apontar um como sempre superior. Assim, para testar a robustez dos resultados, os trabalhos costumam realizar estimações com mais de um método (HECKMAN, 1997, 1998; DEHEJIA; WAHBA, 2002; CALIENDO, KOPEINIG, 2005; SMITH; TODD, 2005).

Neste estudo, optou-se por adotar dois dos métodos mais usados na literatura: i) nearest neighbour matching (NNM) - "vizinho mais próximo"; e ii) Kernel matching (KM). No NNM, cada empresa tratada é pareada à não tratada mais próxima em termos de propensity scores. Pode-se permitir ou não que uma empresa não tratada seja usada mais de uma vez como par de uma firma tratada. Assim, o NNM pode ser com reposição (NNM-CR) ou sem (NNM-SR). É atribuído peso zero às firmas do grupo de controle não pareadas a uma tratada. No NNM-CR, firmas não tratadas pareadas a mais de uma tratada recebem pesos superiores a um, que variam de acordo com o número de vezes em que são utilizados (CALIENDO, KOPEINIG, 2005).

No KM, o contrafactual é construído a partir da média ponderada de todas as empresas do grupo de controle. Os pesos são inversamente proporcionais à distância entre os propensity scores de cada empresa não tratada e a tratada para a qual o contrafactual é calculado. Todas as não tratadas são consideradas, ou seja, não é atribuído o peso zero a nenhuma, nem mesmo

\footnotetext{
${ }^{7}$ Os métodos Logit e Probit são adequados para estimações com variáveis dependentes binárias por ajustarem as probabilidades preditas no intervalo entre 0 e 1 (WOOLDRIDGE, 2002).

${ }^{8}$ Forma proposta por Dehejia e Wahba $(1999,2002)$. Outras formas para impor a condição de suporte comum (ou overlap) e discussões sobre a importância desta condição são apresentadas por Caliendo e Kopeinig (2005), Smith e Todd (2005), Heckman et al. (1997, 1998, 1999), entre outros.

${ }^{9}$ Em conjunto, estas são chamadas de strong ignorability assumption (ROSENBAUM; RUBIN, 1983).
} 
àquelas bem diferentes das tratadas. Por isso, além do suporte comum, pode-se impor uma distância máxima (bandwidth) entre os propensity scores das tratadas e não tratadas. Seguindo a prática da literatura, são feitas estimações com bandwidth de 0,06 e de 0,01 (HECKMAN, 1997, 1998; SMITH; TODD, 2005). Apesar da literatura não apontar um método de PSM como superior aos demais, Caliendo e Kopeining (2005) sugerem que o KM é preferível ao NNM se o grupo dos tratados possuir poucas observações, como ocorre neste estudo (Tabelas 1 e 2).

Para verificar a robustez dos resultados, neste estudo também são estimados modelos por Mínimos Quadrados Ordinários (MQO) - com erros-padrão robustos ${ }^{10}-$, adotando as seguintes estratégias: i) produtividade do trabalho e PTF como variáveis dependentes $\left(Y_{i}\right)$; ii) dummy insuficiência de água $\left(A_{i}\right)$ como variável explicativa de interesse; iii) mesmas variáveis do PSM como controles $\left(X_{i}\right)$; e ii) observações no suporte comum e ponderadas pelos propensity scores resultantes de cada um dos 4 métodos de matching aqui empregados. Ao contrário dos métodos de PSM, o MQO no suporte comum e ponderado impõe forma funcional aditiva, mas sempre é importante combinar métodos para obter maior precisão às estimações (FIRPO; PINTO, 2013). Ademais, também são realizadas estimações por MQO tradicional - erros-padrão robustos, mesmas variáveis de controle $X_{i}$, sem ponderação e sem imposição do suporte comum.

Resumidamente, neste estudo, pelos métodos apontados, são estimados modelos em que as variáveis dependentes $\left(Y_{i}\right)$ são: i) produtividade do trabalho $-\ln \left(\operatorname{Prod} L_{i}\right)=\ln \left(V_{i} / L_{i}\right)$-; e ii) PTF - resíduo $\left(\varepsilon_{i}\right)$ da estimação da equação (1) por MQO (erros-padrão robustos ${ }^{11}$ ). A Tabela 3 apresenta médias e desvios-padrão das variáveis dependentes, segundo as amostras nas quais são consideradas. A variável explicativa de interesse $\left(A_{i}\right)$ é a dummy insuficiência de água.

Tabela 3 - Variáveis dependentes $\left(Y_{i}\right)$ : estatísticas descritivas, segundo as amostras

\begin{tabular}{c|c|c|c}
\hline Amostras & Variáveis & Médias & Desvios-Padrão \\
\hline \multirow{3}{*}{ Amostra I } & Vendas $\left(V_{i}\right)$ & $22.222 .945,56$ & $96.825 .701,42$ \\
& Trabalho $\left(L_{i}\right)$ & 151,70 & 617,88 \\
\cline { 2 - 4 } & Produtividade Trabalho $\left(V_{i} / L_{i}\right)$ & $273.658,83$ & $3.281 .642,13$ \\
\hline \multirow{3}{*}{ Amostra II } & Vendas $\left(V_{i}\right)$ & $24.029 .602,43$ & $105.889 .349,37$ \\
& Capital $\left(K_{i}\right)$ & $8.656 .534,67$ & $72.161 .321,22$ \\
\hline & Trabalho $\left(L_{i}\right)$ & 162,52 & 678,54 \\
\hline & Insumos Intermediários $\left(M_{i}\right)$ & $6.949 .414,84$ & $30.130 .882,71$ \\
\hline
\end{tabular}

Fonte: Banco Mundial, ICS. Elaboração própria.

O Quadro 1 mostra as variáveis de controle $X_{i}$, todas oriundas da ICS, divididas em três grupos. O primeiro representa características das firmas; o segundo é composto por atributos dos trabalhadores/trabalho; e o terceiro corresponde a proxies para o desempenho produtivo. Além disso, também são controladas dummies setoriais - para os setores da Tabela 2 (alimentos como default) e dummies estaduais - para os estados da Tabela 1 (São Paulo como default).

As variáveis explicativas de controle $\left(X_{i}\right)$ foram escolhidas, respeitando a disponibilidade de dados, por representarem, segundo a literatura, possíveis determinantes da produtividade e, provavelmente, da insuficiência de água. O tempo que o gerente principal trabalha no setor e a idade da firma podem influenciar a produtividade por meio de learning by doing - aprendizado acumulado que gera práticas benéficas (SYVERSON, 2011). Banco Mundial (2008) defende imperfeições dos mercados de crédito e capital como fontes de vantagens significativas para as grandes empresas frente às pequenas e médias, o que justifica a variável trabalhadores como proxy para o tamanho da empresa. Paula (2017) mostra a dificuldade de pequenas e médias

\footnotetext{
${ }^{10}$ Erros-padrão pela matriz proposta por White (1980).

${ }^{11}$ Pela matriz de White (1980), conforme é proposto por Escribano e Guasch (2008).
} 
empresas acessarem o mercado de crédito brasileiro. O market-share (proxy mercado) também pode gerar vantagens nos mercados de crédito e capital, com reflexos sobre a produtividade.

Quadro 1 - Variáveis explicativas de controle $X_{i}$ : descrições

\begin{tabular}{|c|c|c|}
\hline Grupos & Variáveis & Descrições \\
\hline \multirow{7}{*}{$\begin{array}{c}\text { Características } \\
\text { da Firma }\end{array}$} & Trabalhadores $(\ln )$ & Quantidade de trabalhadores permanentes \\
\hline & Mercado & Participação no mercado (\%) \\
\hline & Gerente & Tempo (anos) que o gerente principal trabalha no setor \\
\hline & Idade & Idade da firma \\
\hline & Exportadora & Dummy firma exportadora \\
\hline & Capital Estrangeiro & Dummy firma com mais de $10 \%$ de capital estrangeiro \\
\hline & Certificação & Dummy firma com certificação internacional \\
\hline \multirow{2}{*}{$\begin{array}{l}\text { Características dos } \\
\text { Trabalhadores/Trabalho }\end{array}$} & $3^{\circ}$ grau & Proporção de trabalhadores com terceiro grau (\%) \\
\hline & Treinamento & Dummy firma com treinamento \\
\hline \multirow{2}{*}{$\begin{array}{l}\text { Desempenho } \\
\text { Produtivo }\end{array}$} & NUCI & Nível de utilização da capacidade instalada (\%) \\
\hline & Turno & Horas trabalhadas por turno \\
\hline
\end{tabular}

Fonte: Banco Mundial, ICS. Elaboração própria.

A proporção de trabalhadores com $3^{\circ}$ grau e a existência de treinamento na firma captam a "qualidade da mão-de-obra" - seu capital humano. Trabalhadores com maior capital humano tendem a ser mais produtivos, o que afeta diretamente a produtividade do trabalho e a PTF. Sharpe (2003) aponta este como um dos principais drivers do crescimento da produtividade.

A empresa ser exportadora pode afetar a produtividade devido à exposição à concorrência internacional. Nesse sentido, a certificação internacional é uma proxy para a excelência em processos produtivos. Isto porque, ter certificação pressupõe, em algum grau, possuir padrões produtivos de acordo com as "melhores práticas", que podem significar o uso mais eficiente da força de trabalho e do capital. $\mathrm{O}$ uso da capacidade instalada relaciona-se com a produtividade devido a possíveis efeitos de escala. As horas trabalhadas por turno pode ter relação decrescente com a produtividade do trabalho (CAVALCANTE; DE NEGRI, 2014).

Pelo lado da insuficiência de água, é plausível supor que tamanho e market share possam garantir mais incentivos e recursos para a empresa evitar e contornar interrupções de água, com o uso de fontes alternativas, por exemplo. Idade, experiência do gestor e trabalhadores treinados e mais especializados podem garantir expertise para a empresa lidar com problemas de acesso a água. Nesse sentido, podem contribuir exportação e certificação internacional, indicadores de excelência de planejamento e organização, pois o primeiro leva a importantes aprendizados e o segundo é uma proxy para o reconhecimento. Quanto maiores a capacidade instalada e as horas trabalhadas, maior tende a ser a necessidade por água, o que pode afetar a insuficiência de água.

Quanto às dummies setoriais, Moyo (2014) mostra que setores com maior uso de água na produção são mais afetados por sua escassez. As dummies dos estados brasileiros buscam captar diferenças existentes no abastecimento de água, em termos de quantidade e qualidade, entre os estados brasileiros (ANA, 2010; IBGE, 2011; SAIANI et al., 2015); assim como de outros tipos de infraestrutura (IPEA, 2010) que também afetam a produtividade. A análise de empresas de um país permite que sejam avaliados efeitos na produtividade em um mesmo contexto macro institucional; já as dummies estaduais também podem captar diferenças institucionais estaduais.

Por último, deve-se apontar que os propensity scores para os pareamentos são calculados pela estimação da equação (6) pelo método Logit. Uma discussão dos coeficientes, mostrados nas Tabelas A.1 (amostra 1) e A.2 (amostra II) do Apêndice, foge do escopo deste estudo. Ressalva-se apenas que há variáveis não significativas. Segundo, Bryson et al. (2002) a inclusão de variáveis não significativas no PSM não viesa as estimações. Já Caliendo e Kopeinig (2005) apontam que uma variável só deve ser excluída se houver consenso de que não afeta o resultado.

$\operatorname{Prob}\left(A_{i}=1 \mid X_{i}\right)=P\left(X_{i}\right)=\mu_{0}+\mu_{1} X_{i}+e_{i}$ 
sendo: $A_{i}$ a dummy insuficiência de água; $\mu_{0}$ a constante; $X_{i}$ o vetor de variáveis explicativas da empresa $i$ (Quadro 1 e dummies setoriais e estaduais); $\mu_{1}$ o vetor de coeficientes associados às variáveis explicativas; e $e_{i} \mathrm{o}$ erro.

Para avaliar a qualidade dos pareamentos, segue-se Rosenbaum e Rubin (1985): testes t de diferenças de médias entre os grupos de tratamento e controle para cada variável explicativa após o matching. Os testes são feitos para as empresas no suporte comum, de acordo com Lee (2004). A hipótese nula é que as diferenças das médias sejam nulas. Se as estatísticas t são significativas, as médias são estatisticamente diferentes. Com o pareamento, diferenças das médias entre os grupos devem sumir. Tais testes também são mostrados nas Tabelas A.1 e A.2 do Apêndice - amostras I e II, respectivamente - para todos os métodos de PSM adotados. Observa-se que, no geral, as estatísticas t não são significativas, o que sinaliza que os grupos estão balanceados, ou seja, são comparáveis em termos dos atributos observados considerados.

\section{Análise dos resultados}

A seguir, são analisados os resultados das estimações que permitem avaliar os efeitos da insuficiência de água na produtividade do trabalho e na PTF de empresas brasileiras. Para não fugir do escopo do presente estudo, os coeficientes associados às variáveis de controle não são reportados nas estimações em que isso é possível. Estes podem ser obtidos junto aos autores.

A Tabela 4 apresenta os resultados das estimações por MQO tradicional, com e sem as dummies setoriais e estaduais - especificações $I$ e $I I$, respectivamente. Para a produtividade do trabalho, a insuficiência de água é associada a efeito médio negativo e significativo (5\%) apenas na especificação I, sinalizando a relevância da inclusão das dummies setoriais e estaduais ${ }^{12}$. Como a produtividade do trabalho é considerada nas estimações em logaritmo, o coeficiente sugere redução média de $31,3 \%$ nesta produtividade nas empresas com insuficiência de água ${ }^{13}$.

Tabela 4 - MQO: resultados

\begin{tabular}{c|c|c|c|c}
\hline Variáveis / Estatísticas / & \multicolumn{2}{|c|}{ Produtividade do Trabalho } & \multicolumn{2}{c}{ PTF } \\
\cline { 2 - 5 } Especificações & I & II & I & II \\
\hline Insuficiência de Água & $-0,376^{*}$ & $-0,368$ & $-0,420^{* * *}$ & $-0,414 * *$ \\
& $(0,228)$ & $(0,248)$ & $(0,152)$ & $(0,153)$ \\
\hline Variáveis de Controle & Sim & Sim & Sim & Sim \\
Dummies Setoriais & Sim & Não & Sim & Não \\
Dummies Estaduais & Sim & Não & Sim & Não \\
\hline$R^{2}$ & 0,330 & 0,143 & 0,129 & 0,093 \\
Observações & 982 & 982 & 743 & 743 \\
\hline
\end{tabular}

Erros-padrão robustos entre parênteses. *** Significativo a 1\%. ** Significativo a 5\%. * Significativo a $10 \%$.

Na PTF, os efeitos médios, estimados por MQO tradicional, da dummy insuficiência de água são negativos e significativos (1\%) nas duas especificações. Como é considerado a PTF em logaritmo, na especificação I, o efeito representa uma redução média da PTF de 31,4\% nas empresas que sofreram falta de água; na especificação II, a redução média da PTF é de 33,9\%. Os efeitos médios significativos na PTF são, portanto, maiores que o efeito médio significativo sobre a produtividade do trabalho, o que pode sugerir que a insuficiência de água pode impactar sobre os outros insumos de produção (capital e intermediários) e não somente sobre o trabalho.

\footnotetext{
${ }^{12}$ Provavelmente devido a aspectos já comentados: i) utilização de água na produção ser diferente entre os setores; e ii) problemas no abastecimento de água divergirem entre os estados.

${ }^{13} \mathrm{O}$ efeito de uma dummy em variável dependente em logaritmo é calculado pela expressão: $100[\exp (\hat{\beta})-1]$, sendo $\hat{\beta}$ o coeficiente estimado da dummy (WOOLDRIDGE, 2002). Tal cálculo é adotado em todas as análises.
} 
A Tabela 5 mostra os resultados pelos métodos de PSM para a produtividade do trabalho. Observa-se que, tanto pelo método de nearest neighbour matching - com reposição (NNM$\mathrm{CR}$ ) ou sem (NNM-SR) -, como por Kernel matching (KM)- com bandwidth de 0,06 ou 0,01 -, os efeitos médios estimados associados à insuficiência de água são negativos, mas nenhum é significativo, ao contrário do efeito negativo e significativo da especificação I do MQO, o que pode ser explicado pelo possível viés de auto seleção, justificando o emprego de $\operatorname{PSM}^{14}$.

Tabela 5 - PSM: resultados (amostra I)

\begin{tabular}{c|c|c|c|c}
\hline Variáveis / Estatísticas / & \multicolumn{4}{|c}{ Produtividade do Trabalho } \\
\cline { 2 - 5 } Métodos & NNM-CR & NNM-SR & KM (0,06) & KM (0,01) \\
\hline Insuficiência de Água & $-0,327$ & $-0,437$ & $-0,326$ & $-0,261$ \\
Estatísticas t & $(0,352)$ & $(0,330)$ & $(0,254)$ & $(0,277)$ \\
\hline Observações: tratamento/controle & $-0,93$ & $-1,33$ & $-1,28$ & $-0,94$ \\
\hline
\end{tabular}

Erros-padrão entre parênteses. *** Significativo a 1\%.* Significativo a 5\%. * Significativo a $10 \%$.

Já na PTF, a Tabela 6 mostra que os efeitos médios da insuficiência de água estimados por PSM, assim como por MQO, são negativos e significativos. Por NNM-CR e KM com bandwidth de 0,06, o efeito é uma redução média de 33,2\% da PTF nas empresas que sofreram falta de água. Por NNM-SR, o efeito é de uma redução média da PTF de $39 \%$ nas empresas com insuficiência de água; já por KM com bandwidth de 0,01 , a redução média é de 46,4\%.

Tabela 6 - PSM: resultados (amostra II)

\begin{tabular}{c|c|c|c|c}
\hline Variáveis / Estatísticas / & \multicolumn{4}{|c}{ PTF } \\
\cline { 2 - 5 } Métodos & NNM-CR & NNM-SR & KM (0,06) & KM (0,01) \\
\hline \multirow{2}{*}{ Insuficiência de Água } & $-0,332^{*}$ & $-0,339^{*}$ & $-0,332^{* *}$ & $-0,464 * * *$ \\
& $(0,188)$ & $(0,181)$ & $(0,155)$ & $(0,179)$ \\
\hline Estatísticas t & $-1,76$ & $-1,87$ & $-2,14$ & $-2,60$ \\
Observações: tratamento/controle & $44 / 44$ & $44 / 44$ & $43 / 699$ & $43 / 699$ \\
\hline
\end{tabular}

Erros-padrão entre parênteses. $* * *$ Significativo a $1 \%$. ** Significativo a $5 \%$ * Significativo a $10 \%$.

A Tabela 7 mostra, para a produtividade do trabalho, os resultados por MQO ponderado pelos scores dos métodos de PSM e com a imposição do suporte comum. A combinação dos métodos pode garantir maior precisão às estimações (FIRPO; PINTO, 2013). A insuficiência de água, em todos os métodos de PSM, é associada a efeitos negativos não significativos sobre a produtividade do trabalho, o que está em consonância com a maioria dos resultados anteriores

Tabela 9 - MQO ponderado: resultados (amostra I)

\begin{tabular}{c|c|c|c|c}
\hline Variáveis / Estatísticas / & \multicolumn{4}{|c}{ Produtividade do Trabalho } \\
Métodos & NNM-CR & NNM-SR & KM (0,06) & KM (0,01) \\
\cline { 2 - 5 } Insuficiência de Água & $-0,200$ & $-0,332$ & $-0,277$ & $-0,248$ \\
& $(0,320)$ & $(0,300)$ & $(0,195)$ & $(0,226)$ \\
Variáveis de Controle & Sim & Sim & Sim & Sim \\
Dummies Setoriais e Estaduais & Sim & Sim & Sim & Sim \\
\hline$R^{2}$ & 0,301 & 0,249 & 0,344 & 0,368 \\
Observações & 109 & 118 & 977 & 946 \\
\hline
\end{tabular}

Erros-padrão robustos entre parênteses. *** Significativo a 1\%. ** Significativo a 5\%. * Significativo a $10 \%$.

A Tabela 8 mostra os resultados por MQO ponderado para a PTF. A insuficiência de água é associada a efeitos médios negativos sobre a PTF. O efeito não é significativo somente na

\footnotetext{
${ }^{14}$ Por KM, os erros-padrão são calculados convencionalmente. Para robustez, poderia ser utilizado bootstrap, mas Abadie e Imbens (2008) apontam problemas nessa estratégia. Por NNM, adota-se a proposta de Abadie et al. (2004) de variância condicional robusta à heterocedasticidade.
} 
estimação por NNM-CR. Pela ponderação a partir do KM, que pode ser mais adequado neste estudo devido ao pequeno número de observações dos tratados (CALIENDO; KOPEINING, 2005), com bandwidth é de 0,06 , o efeito é uma redução média de $28,17 \%$ da PTF nas empresas que sofreram insuficiência de água para a produção; já com bandwidth de 0,01 , a redução média é de $32,02 \%$. Pela ponderação a partir do método NNM-SR, a redução média nas é de 26,8\%.

Tabela 8 - MQO ponderado: resultados (amostra II)

\begin{tabular}{c|c|c|c|c}
\hline Variáveis / Estatísticas / & \multicolumn{4}{c}{ PTF } \\
\cline { 2 - 5 } Métodos & NNM-CR & NNM-SR & KM (0,06) & KM (0,01) \\
\hline \multirow{2}{*}{ Insuficiência de Água } & $-0,255$ & $-0,312^{*}$ & $-0,331^{* * *}$ & $-0,386^{* * *}$ \\
& $(0,196)$ & $(0,184)$ & $(0,116)$ & $(0,111)$ \\
Variáveis de Controle & Sim & Sim & Sim & Sim \\
Dummies Setoriais e Estaduais & Sim & Sim & Sim & Sim \\
\hline$R^{2}$ & 0,368 & 0,252 & 0,193 & 0,214 \\
Observações & 80 & 88 & 742 & 708 \\
\hline
\end{tabular}

Erros-padrão robustos entre parênteses. *** Significativo a 1\%.** Significativo a 5\%. * Significativo a $10 \%$.

Portanto, os resultados obtidos nas diferentes estimações permitem interpretar, com certa robustez, como causal o efeito médio negativo da insuficiência de água para a produção sobre a PTF da amostra de empresas manufatureiras com informações disponibilizadas pelo banco de dados ICS do Banco Mundial ${ }^{15}$. O efeito é sensível ao método utilizado - variando de $26,8 \%$ e $46 \%$-, mas mantendo sempre o sinal negativo e sendo significativo na maioria das estimações.

A aparente ausência de efeito significativo na produtividade do trabalho pode sinalizar dois aspectos. Primeiro, que a insuficiência de água afeta a PTF devido a efeitos sobre outros insumos (capital e intermediários). Segundo, que problemas no abastecimento de água geram impactos na produtividade dos trabalhadores, via saúde, como defende a literatura apontada na segunda seção, provavelmente quando estes ocorrem em seus domicílios na água para consumo ou em contaminações no entorno destes, não em função de insuficiência de água nas empresas.

\section{Considerações finais}

Incrementos na produtividade podem garantir o crescimento de uma economia, no curto e longo prazo, com reduções de desigualdades. Trabalhos recentes mostram que a produtividade no Brasil é baixa e avança aquém do necessário. As causas de tais problemas motivam amplos debates e controvérsias no campo econômico. Porém, é consensual na literatura a importância das infraestruturas econômicas como determinantes da produtividade do trabalho e da PTF.

O Brasil, devido a investimentos históricos insuficientes, entre outros fatos, possui sérios gargalos referentes às infraestruturas, dentre as quais, o abastecimento de água. Este, mesmo gerando externalidades positivas, ainda tem graves problemas, como indisponibilidade hídrica ou racionamentos em vários locais, déficits de acesso distribuídos desigualmente, perdas na distribuição e baixa qualidade de parte da água ofertada. Tais problemas afetam as empresas. A intermitência no fornecimento de água pode interromper ou reduzir os processos produtivos, criar dificuldades de comercialização, contaminar a produção e gerar custos adicionais ao forçar as empresas a buscarem fontes alternativas, fatores estes que podem influenciar a produtividade.

Considerando tais aspectos e a existência de poucas evidências nesse sentido no nível das empresas, pelo menos para o Brasil, este estudo avaliou, empiricamente, se a insuficiência de água para a produção influencia a produtividade do trabalho e a PTF das empresas brasileiras manufatureiras com informações disponibilizadas pela base ICS do Banco Mundial. Para isso,

${ }^{15}$ Evidências similares foram obtidas por Moyo (2011) para países africanos com dados do ICS. 
foram adotadas estratégias recomendadas na literatura e utilizados alguns métodos de estimação por Propensity Score Matching (PSM) para lidar com a possibilidade de viés de auto seleção.

Os resultados encontrados sugerem, com certa robustez, reduções médias da PTF devido à insuficiência de água nas empresas da amostra que passaram por tal problema em 2007. Ou seja, pode-se inferir que problemas no abastecimento de água têm efeitos médios negativos na PTF das empresas brasileiras. Portanto, tal evidência contribui para o preenchimento de uma lacuna na literatura, corroborando discussões e resultados de outros trabalhos. Na produtividade do trabalho, a insuficiência de água não foi associada, no geral, a efeitos significativos, o que sugere que problemas no abastecimento de água afetam esta produtividade, via saúde, como aponta a literatura, quando estes ocorrem nos (ou perto dos) domicílios dos trabalhadores.

\section{Referências bibliográficas}

ABADIE, A.; DRUKKER, D.; HERR, J. L.; IMBENS, G. W. "Implementing matching estimators for average treatment effects in Stata". Stata Journal, n.4, 2004.

ABADIE, A.; IMBENS, G. W. "On the failure of the bootstrap for matching estimators". Econometrica, v.76, n.6, 2008.

ANA. Atlas Brasil: abastecimento urbano de água. ANA, 2010.

ANA. Água na indústria: uso e coeficientes técnicos. ANA, 2017.

ASCHAUER, D. "Is public expenditure productive?" Journal of Monetary Economics, v.23, n.2, 1989.

BANCO MUNDIAL. Relatório sobre o desenvolvimento mundial 1994: infraestrutura para o desenvolvimento. Banco Mundial, 1994.

BANCO MUNDIAL. Conhecimento e inovação para a competitividade. Banco Mundial, 2008. BARRO, R. J. "Government spending in a simple model of endogeneous growth". The Journal of Political Economy, v.98, n.5, 1990.

BREGMAN, A.; MAROM, A. Productivity factors in Israel's manufacturing industry. Bank of Israel Economic Review, n.72, 1999.

BRYSON, A.; DORSETT, R.; PURDON, S. "The use of propensity score matching in the evaluation of labour market policies". Working Paper of Department for Work and Pensions, n.4, 2002.

CALIENDO, M.; KOPEINIG, S. "Some practical guidance for the implementation of propensity score matching". IZA Discussion Papers, n.1588, IZA, 2005.

CAVALCANTE, L. R.; DE NEGRI, F. "Evolução recente dos indicadores de produtividade no Brasil". In: DE NEGRI, F.; CAVALCANTE, L. R. Produtividade no Brasil: desempenho e determinantes - desempenho, v.1, IPEA, 2014.

COX, D. R. Planning of experiments. Wiley, 1958.

CRUZ, A. C.; TEIXEIRA, E. C.; BRAGA, M. J. "Os efeitos dos gastos públicos em infraestrutura e em capital humano no crescimento econômico e na redução da pobreza no Brasil". EconomiA, v.11, n.4, 2010.

CVJETANOVIC, B. "Health effects and impact of water supply and sanitation". World Health Statistics Quarterly, v.39, 1986.

DE NEGRI, F.; CAVALCANTE, L. R. "Os dilemas e desafios da produtividade no Brasil". In: DE NEGRI, F.; CAVALCANTE, L. R. Produtividade no Brasil: desempenho e determinantes - desempenho, v.1., IPEA, Brasília, 2014a.

DE NEGRI, F.; CAVALCANTE, L. R. Produtividade no Brasil: desempenho e determinantes - desempenho, v.1 e 2, IPEA, Brasília, 2014b. 
DUFLO, E.; GLENNERSTER, R.; KREMER, M. "Using randomization in development economics research: a toolkit". MIT Department of Economics Working Paper, n.6, v.36, 2006. EASTERLY, W. REBELO, S. "Fiscal policy and economic growth: an empirical investigation". Journal of Monetary Economics, v.32, 1993.

ELLERY JÚNIOR, R. "Desafios para o cálculo da produtividade total dos fatores". In: DE NEGRI, F.; CAVALCANTE, L. R. Produtividade no Brasil: desempenho e determinantes desempenho, v.1., IPEA, Brasília, 2014.

ESCRIBANO, A.; GUASCH, J. L. Robust methodology for investment climate assessment on productivity: application to Investment Climate Surveys from Central America. Working Paper, Economic Series, Universidad Carlos III, 2008.

ESCRIBANO, A.; GUASCH, J. L.; ORTE, M.; PENA, J. Investment climate assessment based on demean olley and pakes decompositions: methodology and application to Turkey's Investment Climate Survey. Working Paper, Economic Series, Universidad Carlos III, 2008.

ESCRIBANO, A.; GUASCH, J. L.; PENA, J. "Assessing the impact of infrastructure quality on firm productivity in Africa". Policy Research Working Paper, v.5191, 2010.

FAJNZYLBER, P.; GUASCH, J. L.; LÓPEZ, J. H. Does the investment climate matter? Microeconomic foundations of growth in Latin America. MacMillan, 2008.

FERREIRA, P. C. "Investimento em infraestrutura no Brasil: fatos estilizados e relações de longo prazo". Pesquisa e Planejamento Econômico, v.26, n.2, 1996.

FERREIRA, P. C. G.; MALLIAGROS, T. G. "O impacto da infraestrutura sobre o crescimento da produtividade do setor privado e do produto brasileiro". Ensaios Econômicos, n.315, 1997. FIRPO, S. P.; PINTO, R. C. C. "Combinando estratégias para estimação de efeitos de tratamento". Working Paper EESP, n.3, 2013.

HECKMAN, J.; ICHIMURA, H.; TODD, P. "Matching as an econometric evaluation estimator: evidence from evaluating a job training programme". Review of Economic Studies, v.64, n.4, 1997.

HECKMAN, J.; ICHIMURA, H.; SMITH, J.; TODD, P. “Characterizing selection bias using experimental data". Econometrica, v.66, n.5, 1998.

HECKMAN, J.; LALONDE, R.; SMITH, J. "The economics and econometrics of active labor market programs". In: ASHENFELTER, O.; CARD, D. (Eds.). The Handbook of Labor Economics. North Holland, Amsterdam, 1999.

HELLER, L. Saneamento e saúde. Organização Pan-Americana da Saúde, 1997.

IBGE. Atlas de Saneamento 2011. IBGE, Rio de Janeiro, 2011.

IBRE. Benefícios econômicos da expansão do saneamento brasileiro. Trata Brasil, IBRE, 2010

IPEA. Infraestrutura econômica no Brasil: diagnósticos e perspectivas para 2025. IPEA, 2010. LEE, W. S. "Propensity score matching and variations in the balancing test". Empirical Economics, v.44, n.1, 2006.

MASTROMARCO, C.; WOITEK, W. "Public infrastructure investment and efficiency in Italian regions". Journal of Productivity Analysis, v. 25, n.1-2, 2006.

MESSA, A. "Metodologias de cálculo da produtividade total dos fatores e da produtividade da mão de obra". In: DE NEGRI, F.; CAVALCANTE, L. R. Produtividade no Brasil: desempenho e determinantes - desempenho, v.1., IPEA, 2014.

MIGUEZ, T. MORAES, T. "Produtividade do trabalho e mudança estrutural: uma comparação internacional com base no World Input-Output Database (WIOD) 1995-2009”. In: DE NEGRI, F.; CAVAlCANTE, L. R. Produtividade no Brasil: desempenho e determinantes desempenho, v.1., IPEA, 2014.

MOYO, B. "Do water cuts affect productivity? Case study of African manufacturing firms". Water SA, v.37, n.3, 2011. 
MORRISON, C. P.; SCHWARTZ, A. "State infrastructure and productive performance". American Economic Review, v.86, n.5, 1996.

MUNNEL, A. H. "Why has productivity declined? Productivity and public investment". New England Economic Review, 1990.

MUSSOLINI, C.; TELES, V. K. "Infraestrutura e produtividade no Brasil". Revista de Economia Política, v.30, n.4, 2010.

PAULA, G. Inclusão financeira de pequenas e médias empresas no Brasil. CEPAL, 2017.

PÊGO FILHO, B.; CÂNDIDO JÚNIOR, J. O.; PEREIRA, F. "Investimento e financiamento da infraestrutura no Brasil: 1990/2002”. Textos para Discussão do IPEA, n.680, 1999.

REZENDE, S. C; WAJNMAN, S.; CARVALHO, J. A. M.; HELLER, L. "Integrando oferta e demanda de serviços de saneamento: análise hierárquica do panorama urbano brasileiro no ano 2000”. Revista de Engenharia Sanitária Ambiental, v.12, n.1, 2007.

RIGOLON, F. J. Z. "O investimento em infraestrutura e a retomada do crescimento econômico sustentado". Pesquisa e Planejamento Econômico, v.28, n. 1, 1998.

ROSENBAUM, P. R.; RUBIN, D. B. "Constructing a control group using multivariate matched sampling methods that incorporate the propensity score". The American Statistician, n.39, 1985.

ROY, A. D. "Some thoughts on the distribution of earnings". Oxford Economic Papers, v.3, n.2, 1951.

RUBIN, D. B. "Estimating causal effects of treatments in randomized and nonrandomized studies". Journal of Educational Psychology, v.66, n.5, 1974.

RUBIN, D. B. "Assignment of treatment group on the basis of a covariate". Journal of Educational and Behavioral Statistics, n.2, 1977.

RUBIN, D. B. "Bayesian inference for causal effects: the role of randomization". The Annals of Statistics, v.6, n.1, 1978.

SMITH, J. A.; TODD, P. E. “Does matching overcome LaLonde's critique of nonexperimental estimators?". Journal of Econometrics, Elsevier, n.125, 2005.

SAIANI, C. C. S.; RODRIGUES, R. L.; GALVÃO, G. C. Saneamento básico no Brasil e Objetivos de Desenvolvimento do Milênio: evoluções dos déficits de acesso de 1990 a 2010. Cadernos de Ciências Sociais Aplicadas, v.12, 2015.

SHARPE, A. "Why are Americans more productive than Canadians? International Productivity Monitor, n.6, 2003.

SCHETTINI, D.; AZZONI, C. "Determinantes regionais da produtividade industrial: o papel da infraestrutura". In: DE NEGRI, F.; CAVALCANTE, L. R. Produtividade no Brasil: desempenho e determinantes - desempenho, v.1, IPEA, 2014.

SYVERSON, C. "What determines productivity?. Journal of Economic Literature, v.49, n.2, 2011.

TONETO JÚNIOR, R.; SAIANI, C. C. S. "Restrições à expansão dos investimentos no saneamento básico brasileiro". Revista Econômica do Nordeste, v.37, 2006.

TUROLLA, F. A. "Política de saneamento básico: avanços recentes e opções futuras de políticas públicas". Textos para Discussão do IPEA, Brasília, n.922, 2002.

UCHIMURA, K.; GAO, H. The importance of infrastructure on economic development. World Bank, Latin America and the Caribbean Regional Office, Washington, 1993.

WHITE, H. "A heteroskedasticity-consistent covariance matrix estimator and a direct test for heteroskedasticity". Econometrica, v.48, n.4, 1980.

WOOLDRIDGE, J. M. Introductory econometrics. Pioneira Thomson Learning, 20ed., 2002. 


\section{Apêndice}

Tabela A.1 - Amostra 1 (produtividade do trabalho): resultados (Logit) e testes de diferenças de médias, segundo os métodos de PSM

\begin{tabular}{|c|c|c|c|c|c|c|c|c|c|c|}
\hline \multirow{3}{*}{ Variáveis } & \multirow{3}{*}{ Coeficientes } & \multirow{3}{*}{$\begin{array}{c}\begin{array}{c}\text { Grupo de } \\
\text { Tratamento }\end{array} \\
\text { Médias } \\
\end{array}$} & \multicolumn{8}{|c|}{ Grupo de Controle } \\
\hline & & & \multicolumn{2}{|c|}{ NNM-CR } & \multicolumn{2}{|c|}{ NNM-SR } & \multicolumn{2}{|c|}{ KM (bandwidth: 0,06) } & \multicolumn{2}{|c|}{ KM (bandwidth: 0,01$)$} \\
\hline & & & Médias & Estatísticas t & Médias & Estatísticas t & Médias & Estatísticas t & Médias & Estatísticas t \\
\hline Trabalhadores & $-0,432 * *$ & 3,388 & 3,470 & 0,682 & 3,298 & 0,675 & 3,398 & 0,963 & 3,386 & 0,993 \\
\hline Mercado & 6,130 & 0,005 & 0,005 & 0,993 & 0,005 & 0,986 & 0,004 & 0,954 & 0,003 & 0,763 \\
\hline Gerente & 0,011 & 22,760 & 23,880 & 0,626 & 23,190 & 0,852 & 22,560 & 0,928 & 21,950 & 0,716 \\
\hline Idade & 0,005 & 20,560 & 21,710 & 0,720 & 21,490 & 0,772 & 20,390 & 0,961 & 20,160 & 0,904 \\
\hline Exportadora & $-0,378$ & 0,119 & 0,085 & 0,546 & 0,085 & 0,546 & 0,120 & 0,980 & 0,092 & 0,638 \\
\hline Capital Estrangeiro & 0,305 & 0,034 & 0,017 & 0,563 & 0,017 & 0,563 & 0,029 & 0,871 & 0,029 & 0,889 \\
\hline Certificação & $1,079 * * *$ & 0,305 & 0,356 & 0,561 & 0,339 & 0,697 & 0,314 & 0,918 & 0,300 & 0,949 \\
\hline $3^{\circ}$ grau & $-0,023$ & 7,085 & 8,881 & 0,412 & 9,136 & 0,370 & 7,444 & 0,850 & 6,931 & 0,934 \\
\hline Treinamento & $1,223 * * *$ & 0,746 & 0,763 & 0,832 & 0,746 & 1,000 & 0,725 & 0,802 & 0,738 & 0,920 \\
\hline NUCI & 0,000 & 77,150 & 76,200 & 0,774 & 74,420 & 0,453 & 76,920 & 0,947 & 76,860 & 0,933 \\
\hline Turno & $-0,041$ & 7,889 & 8,426 & 0,491 & 7,986 & 0,895 & 7,933 & 0,952 & 8,093 & 0,790 \\
\hline Alimentos & --- & 0,237 & 0,220 & 0,828 & 0,203 & 0,660 & 0,202 & 0,646 & 0,239 & 0,980 \\
\hline Autopeças & $-1,246 * *$ & 0,085 & 0,085 & 1,000 & 0,085 & 1,000 & 0,083 & 0,972 & 0,092 & 0,890 \\
\hline Máquinas e Equipamentos & $-1,424 * *$ & 0,119 & 0,153 & 0,594 & 0,169 & 0,436 & 0,124 & 0,934 & 0,118 & 0,987 \\
\hline Móveis & $-0,895 *$ & 0,153 & 0,085 & 0,259 & 0,085 & 0,259 & 0,147 & 0,935 & 0,151 & 0,985 \\
\hline Químicos & $-0,150$ & 0,220 & 0,305 & 0,300 & 0,305 & 0,300 & 0,241 & 0,792 & 0,223 & 0,969 \\
\hline Sapatos e Couro & $-1,645 * *$ & 0,034 & 0,034 & 1,000 & 0,034 & 1,000 & 0,043 & 0,802 & 0,029 & 0,891 \\
\hline Têxteis & $-2,449 * *$ & 0,017 & 0,000 & 0,319 & 0,000 & 0,319 & 0,033 & 0,581 & 0,020 & 0,910 \\
\hline Outras Manufaturas & 0,067 & 0,017 & 0,051 & 0,313 & 0,034 & 0,563 & 0,019 & 0,929 & 0,023 & 0,825 \\
\hline Vestuário & $-1,052 * *$ & 0,119 & 0,068 & 0,346 & 0,085 & 0,546 & 0,108 & 0,862 & 0,105 & 0,809 \\
\hline Amazonas & $1,754 * *$ & 0,051 & 0,017 & 0,313 & 0,034 & 0,651 & 0,040 & 0,782 & 0,051 & 0,994 \\
\hline Bahia & 0,537 & 0,068 & 0,068 & 1,000 & 0,051 & 0,700 & 0,065 & 0,958 & 0,064 & 0,928 \\
\hline Ceará & 0,639 & 0,051 & 0,051 & 1,000 & 0,051 & 1,000 & 0,053 & 0,950 & 0,071 & 0,655 \\
\hline Goiás & $-0,891$ & 0,017 & 0,017 & 1,000 & 0,017 & 1,000 & 0,019 & 0,929 & 0,016 & 0,972 \\
\hline Mato Grosso & $1,867 * * *$ & 0,085 & 0,068 & 0,731 & 0,068 & 0,731 & 0,062 & 0,640 & 0,084 & 0,981 \\
\hline Minas Gerais & $-0,067$ & 0,085 & 0,102 & 0,754 & 0,085 & 1,000 & 0,086 & 0,984 & 0,088 & 0,944 \\
\hline Paraná & 0,437 & 0,119 & 0,136 & 0,784 & 0,119 & 1,000 & 0,110 & 0,882 & 0,118 & 0,990 \\
\hline Pernambuco & 0,017 & 0,017 & 0,000 & 0,319 & 0,000 & 0,319 & 0,015 & 0,915 & 0,011 & 0,785 \\
\hline Rio de Janeiro & 0,358 & 0,119 & 0,119 & 1,000 & 0,136 & 0,784 & 0,106 & 0,836 & 0,102 & 0,782 \\
\hline Rio Grande do Sul & $-1,385^{*}$ & 0,034 & 0,051 & 0,651 & 0,051 & 0,651 & 0,050 & 0,669 & 0,031 & 0,921 \\
\hline Santa Catarina & 0,571 & 0,136 & 0,153 & 0,795 & 0,136 & 1,000 & 0,155 & 0,768 & 0,135 & 0,989 \\
\hline São Paulo & --- & 0,220 & 0,169 & 0,490 & 0,203 & 0,824 & 0,203 & 0,823 & 0,202 & 0,814 \\
\hline
\end{tabular}

*** Significativo a $1 \%$.** Significativo a $5 \%$. * Significativo a $10 \%$. 
Tabela A.2 - Amostra 2 (PTF): resultados (Logit) e testes de diferenças de médias, segundo os métodos de PSM

\begin{tabular}{|c|c|c|c|c|c|c|c|c|c|c|}
\hline \multirow{3}{*}{ Variáveis } & \multirow{3}{*}{ Coeficientes } & \multirow{3}{*}{$\begin{array}{c}\begin{array}{c}\text { Grupo de } \\
\text { Tratamento }\end{array} \\
\text { Médias } \\
\end{array}$} & \multicolumn{8}{|c|}{ Grupo de Controle } \\
\hline & & & \multicolumn{2}{|c|}{ NNM-CR } & \multicolumn{2}{|c|}{ NNM-SR } & \multicolumn{2}{|c|}{ KM (bandwidth: 0,06) } & \multicolumn{2}{|c|}{ KM (bandwidth: 0,01) } \\
\hline & & & Médias & Estatísticas t & Médias & Estatísticas t & Médias & Estatísticas t & Médias & Estatísticas t \\
\hline Trabalhadores & $-0,520 * * *$ & 3,369 & 3,739 & 0,137 & 3,769 & 0,110 & 3,411 & 0,868 & 3,498 & 0,700 \\
\hline Mercado & 7,947 & 0,006 & 0,006 & 0,985 & 0,006 & 0,960 & 0,005 & 0,911 & 0,006 & 0,950 \\
\hline Gerente & 0,005 & 22,360 & 24,320 & 0,418 & 23,820 & 0,560 & 21,890 & 0,847 & 23,340 & 0,740 \\
\hline Idade & 0,011 & 21,360 & 27,980 & 0,304 & 24,430 & 0,570 & 21,410 & 0,992 & 23,260 & 0,750 \\
\hline Exportadora & $-0,125$ & 0,136 & 0,114 & 0,751 & 0,136 & 1,000 & 0,136 & 1,000 & 0,156 & 0,830 \\
\hline Capital Estrangeiro & 0,472 & 0,045 & 0,045 & 1,000 & 0,045 & 1,000 & 0,043 & 0,956 & 0,053 & 0,900 \\
\hline Certificação & $1,224 * * *$ & 0,318 & 0,341 & 0,823 & 0,364 & 0,660 & 0,295 & 0,817 & 0,357 & 0,760 \\
\hline $3^{\circ}$ grau & $-0,014$ & 8,159 & 7,955 & 0,923 & 7,432 & 0,730 & 8,489 & 0,898 & 10,550 & 0,420 \\
\hline Treinamento & $1,212 * * *$ & 0,750 & 0,773 & 0,805 & 0,727 & 0,810 & 0,721 & 0,761 & 0,748 & 0,970 \\
\hline NUCI & $-0,003$ & 76,750 & 79,430 & 0,464 & 79,160 & 0,510 & 76,980 & 0,956 & 77,980 & 0,670 \\
\hline Turno & $-0,039$ & 8,094 & 7,331 & 0,347 & 7,266 & 0,310 & 7,762 & 0,702 & 7,649 & 0,710 \\
\hline Alimentos & ---- & 0,273 & 0,205 & 0,459 & 0,250 & 0,810 & 0,252 & 0,826 & 0,209 & 0,610 \\
\hline Autopeças & $-1,752 * *$ & 0,045 & 0,114 & 0,242 & 0,091 & 0,400 & 0,049 & 0,938 & 0,050 & 0,950 \\
\hline Máquinas e Equipamentos & $-1,225 * *$ & 0,136 & 0,068 & 0,297 & 0,091 & 0,510 & 0,158 & 0,774 & 0,134 & 0,940 \\
\hline Móveis & $-0,516$ & 0,182 & 0,091 & 0,219 & 0,159 & 0,780 & 0,170 & 0,890 & 0,151 & 0,660 \\
\hline Químicos & 0,625 & 0,023 & 0,068 & 0,312 & 0,023 & 1,000 & 0,027 & 0,891 & 0,048 & 0,540 \\
\hline Sapatos e Couro & $-0,202$ & 0,227 & 0,273 & 0,627 & 0,250 & 0,810 & 0,210 & 0,849 & 0,287 & 0,570 \\
\hline Outras Manufaturas & $-1,945^{*}$ & 0,023 & 0,091 & 0,171 & 0,068 & 0,310 & 0,048 & 0,533 & 0,029 & 0,880 \\
\hline Vestuário & $-1,353 * *$ & 0,091 & 0,091 & 1,000 & 0,068 & 0,700 & 0,085 & 0,928 & 0,094 & 0,990 \\
\hline Amazonas & $2,639 * * *$ & 0,068 & 0,091 & 0,698 & 0,114 & 0,460 & 0,073 & 0,938 & 0,057 & 0,810 \\
\hline Bahia & 0,970 & 0,068 & 0,068 & 1,000 & 0,045 & 0,650 & 0,051 & 0,733 & 0,054 & 0,760 \\
\hline Ceará & $1,479^{*}$ & 0,068 & 0,045 & 0,650 & 0,068 & 1,000 & 0,071 & 0,954 & 0,060 & 0,860 \\
\hline Goiás & $-0,243$ & 0,023 & 0,000 & 0,320 & 0,000 & 0,320 & 0,023 & 0,990 & 0,018 & 0,850 \\
\hline Mato Grosso & $2,078 * *$ & 0,068 & 0,000 & $0,080^{*}$ & 0,000 & $0,080 *$ & 0,045 & 0,645 & 0,020 & 0,490 \\
\hline Minas Gerais & 0,670 & 0,114 & 0,068 & 0,464 & 0,068 & 0,460 & 0,097 & 0,804 & 0,090 & 0,700 \\
\hline Paraná & 0,774 & 0,091 & 0,136 & 0,507 & 0,114 & 0,730 & 0,090 & 0,993 & 0,110 & 0,800 \\
\hline Pernambuco & ---- & --- & --- & --- & --- & --- & --- & --- & --- & --- \\
\hline Rio de Janeiro & 0,826 & 0,136 & 0,182 & 0,565 & 0,182 & 0,570 & 0,138 & 0,977 & 0,155 & 0,840 \\
\hline Rio Grande do Sul & $-0,688$ & 0,045 & 0,000 & 0,156 & 0,000 & 0,160 & 0,062 & 0,734 & 0,040 & 0,880 \\
\hline Santa Catarina & $1,213^{* *}$ & 0,182 & 0,318 & 0,143 & 0,318 & 0,140 & 0,203 & 0,802 & 0,254 & 0,450 \\
\hline São Paulo & ---- & 0,136 & 0,068 & 0,297 & 0,068 & 0,300 & 0,119 & 0,807 & 0,100 & 0,580 \\
\hline
\end{tabular}

*** Significativo a $1 \%$. * Significativo a $5 \%$ * Significativo a $10 \%$. 